\title{
PHARMACOLOGICAL INTERVENTIONS IN FRAILTY AND SARCOPENIA: REPORT BY THE INTERNATIONAL CONFERENCE ON FRAILTY AND SARCOPENIA RESEARCH TASK FORCE
}

\author{
M. CESARI ${ }^{1,2}$, R. FIELDING ${ }^{3}$, O. BÉNICHOU 4 , R. BERNABEI 5 , S. BHASIN 6 , J.M. GURALNIK7, \\ A. JETTE 8 , F. LANDI ${ }^{5}$, M. PAHOR ${ }^{9}$, L. RODRIGUEZ-MANAS $^{10}$, Y. ROLLAND $^{1,2}$, R. ROUBENOFF ${ }^{11}$, \\ A.J. SINCLAIR ${ }^{12}$, S. STUDENSKI ${ }^{13}$, T. TRAVISON ${ }^{14}$, B. VELLAS ${ }^{1,2}$ ON BEHALF OF THE \\ INTERNATIONAL CONFERENCE ON FRAILTY AND SARCOPENIA RESEARCH TASK FORCE
}

\begin{abstract}
1. Gérontopôle, Centre Hospitalier Universitaire de Toulouse, Toulouse, France; 2. INSERM UMR1027, Université de Toulouse III Paul Sabatier, Toulouse, France; 3. Jean Mayer USDA, Human Nutrition Research Center, Boston, MA, USA; 4. Musculoskeletal Area, Eli Lilly \& Co, Paris, France; 5. Department of Geriatrics, Neurosciences, and Orthopedics,

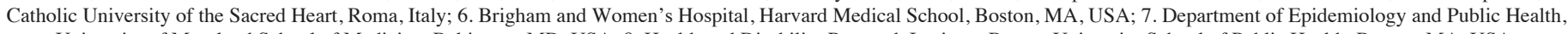
University of Maryland School of Medicine, Baltimore, MD, USA; 8. Health and Disability Research Institute, Boston University School of Public Health, Boston, MA, USA;

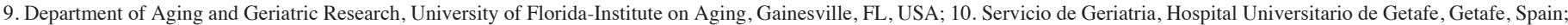
11. Global Translational Medicine, Novartis Institutes for Biomedical Research, Basel, Switzerland; 12. Foundation for Diabetes Research in Older People, Luton, United Kingdom; 13. National Institute on Aging, Baltimore, MD, USA; 14. Institute for Aging Research, Hebrew SeniorLife, Boston, MA, USA

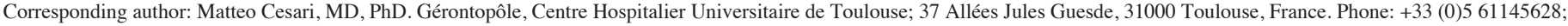
Fax: +33 (0)5 61145640; email: macesari@gmail.com

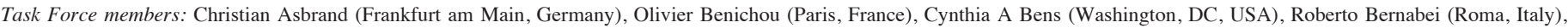

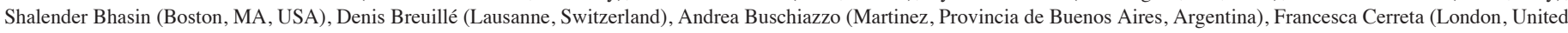

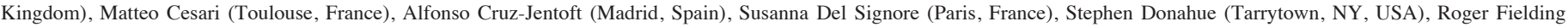

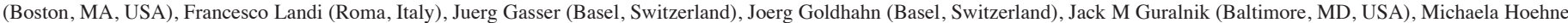

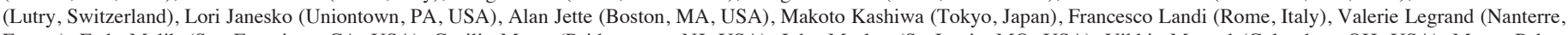

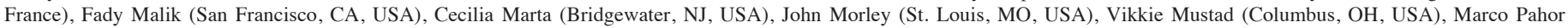

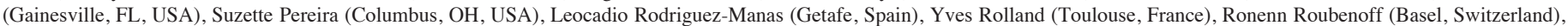

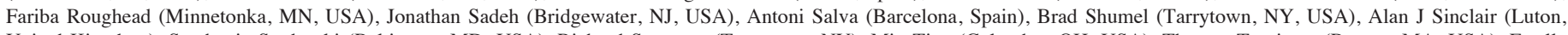

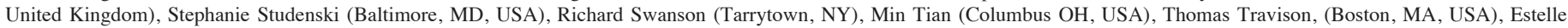
Trifilieff (Basel, Switzerland), Bruno Vellas (Toulouse, France), Sjors Verlaan (Utrecht, The Netherlands), Sander Wijers (Utrecht, The Netherlands).
\end{abstract}

\begin{abstract}
Sarcopenia and frailty often co-exist and both have physical function impairment as a core component. Yet despite the urgency of the problem, the development of pharmaceutical therapies for sarcopenia and frailty has lagged, in part because of the lack of consensus definitions for the two conditions. A task force of clinical and basic researchers, leaders from the pharmaceutical and nutritional industries, and representatives from non-profit organizations was established in 2012 with the aim of addressing specific issues affecting research and clinical activities on frailty and sarcopenia. The task force came together on April 22, 2015 in Boston, Massachusetts, prior to the International Conference on Frailty and Sarcopenia Research (ICFSR). The theme of this meeting was to discuss challenges related to drugs designed to target the biology of frailty and sarcopenia as well as more general questions about designing efficient drug trials for these conditions. The present article reports the results of the task force's deliberations based on available evidence and preliminary results of ongoing activities. Overall, the lack of a consensus definition for sarcopenia and frailty was felt as still present and severely limiting advancements in the field. However, agreement appears to be emerging that low mass alone provides insufficient clinical relevance if not combined with muscle weakness and/or functional impairment. In the next future, it will be important to build consensus on clinically meaningful functional outcomes and test/validate them in long-term observational studies.
\end{abstract}

Key words: Clinical trial, methodology, prevention, disability, physical performance, skeletal muscle.

J Frailty Aging 2015;4(3):114-120

Published online June 24, 2015, http:/ / dx.doi.org/10.14283/jfa.2015.64

\section{Introduction}

Sarcopenia, the age-related loss of muscle mass and strength, represents an increasing public health risk as the world's population ages at a rapid pace (1). Between 2000, and 2050, the United Nations predicts a doubling of the number of people over age 60 (2), and a recent analysis of prevalence studies concluded that sarcopenia prevalence ranges from $1-29 \%$ in community dwelling populations and $14-33 \%$ in long-term care populations (3). Muscle weakness and impaired function that result from sarcopenia are also major components of the geriatric syndrome of frailty (4), thus sarcopenia and frailty are frequently studied in parallel, and indeed they both have physical function impairment as a core condition (5). Yet despite the urgency of the problem, developing treatments for sarcopenia and frailty has lagged, in part because of the lack of consensus definitions for the two conditions.

A task force of clinical and basic researchers, leaders from the pharmaceutical and nutritional industries, and representatives from non-profit organizations came together on April 22, 2015 in Boston, Massachusetts, prior to the International Conference on Frailty and Sarcopenia Research 


\section{THE JOURNAL OF FRAILTY \& AGING}

(ICSFR) to address issues that have slowed the development of new treatments. The increase in size of the Task Force since it was established in 2012 reflects the increasing recognition of the need for more effective ways of treating sarcopenia and frailty, as well as increased attention on the part of industry. This, the third meeting of the Task Force, discussed specific challenges related to drugs designed to target the underlying biology of sarcopenia as well as more general questions about designing efficient drug trials for these conditions.

\section{Targeting the underlying biology of sarcopenia}

Research over the past fifteen years has revealed multiple complex and intersecting pathways involved in the regulation of muscle protein balance as well as many possible approaches to reverse muscle loss. Potential regulators include androgens, which act through androgen receptor/Wnt/beta-catenin signaling pathway; insulin and insulin growth factor 1 (IGF1), which regulate protein synthesis and degradation through the PI3K/AKT pathway; myostatin, a powerful inhibitot of muscle growth, as well as other members of the transforming growth factor- $\beta$ (TGF- $\beta$ ) superfamily, which act through SMAD signaling; and inflammatory modulators including pro-inflammatory cytokines such as tumor necrosis factor- $\alpha$ and interleukin-1 (6). Compounds currently in development for the treatment of muscle wasting and sarcopenia are providing further insight into underlying mechanisms. Examples of two classes of these compounds follow.

\section{Myostatin antagonists}

Bimagrumab is a monoclonal antibody that binds to type II activin receptors (ActRII), blocking the binding of myostatin, GDF11, and activin A. Binding of these ligands normally initiates a signaling cascade that results in decreased muscle growth 7. A single dose of bimagrumab increases muscle mass in healthy young men similar to that achieved with 12 week of high-intensity resistance training $(8,9)$, and in sedentary middle-aged adults, equivalent to that achieved with 9 months of jogging 12-20 miles per week (10). It has also been shown to work in elderly people; and, in a single leg casted model in healthy young men, bimagrumab improved recovery from atrophy.

Novartis received breakthrough therapy approval for bimagrumab for the treatment of sporadic inclusion body myositis (sIBM) in 2013. In people with this rare muscle disease, a single dose of the drug resulted in an increase in 6-minute walking distance (6MWD) of 52 meters over placebo (11), providing the first evidence of a possible clinical benefit. Now the drug is being tested in people in their 70s with low lean muscle mass. Initial results suggest that a single dose of the drug is well tolerated and associated with an increase in appendicular lean mass (aLM) and handgrip strength (12). Interestingly, gait speed was improved only in those with poor results at the baseline $6 \mathrm{MWD}$ test, suggesting that frail people may respond best.

\section{Selective Androgen Receptor Modulators (SARMs)}

Selective androgen receptor modulators (SARMs), a class of androgen receptor ligands that display tissue-selective activation of androgenic signaling, may have potential as function promoting therapies for a variety of conditions, including functional limitations associated with ageing and chronic diseases, osteoporosis, anemia, and hypogonadism, and for male contraception. Osteoporosis represents another component of the frailty syndrome and evidence suggests links between osteoporosis and sarcopenia (13). Indeed, SARMs also have been shown to have favorable effects on bone mass and quality.

A number of steroidal and non-steroidal SARMs have undergone phase I, II and III trials. For instance, the nonsteroidal SARM LGD-4033 has demonstrated preferential tissue selectivity for muscle compared to prostate. Moreover, a 21-day ascending dose study of LGD-4033 in healthy young men showed that the drug was well tolerated, had a favorable pharmacokinetic profile, and increased lean body mass and leg press strength (14).

Another SARM, MK-773, has undergone phase II studies in both men and women with sarcopenia. In one study of women age 65 or older with sarcopenia and frailty, treatment with MK-0773 produced statistically significant increases in LBM compared to placebo, but no significant improvement in strength or function. Both the treatment group and placebo group also received Vitamin D and protein supplementation (15). Other SARMs have also shown benefits in the treatment of muscle wasting associated with cancer, a condition known as cachexia $(16,17)$.

While SARMS appear to be safe and efficacious in increasing LBM and possibly strength and function, their effects on muscle mass and function at the doses that have been studied have been modest in comparison to the effects from treatment with supraphysiologic doses of testosterone (18). It is possible that longer studies are needed to demonstrate functional improvements, but it is also likely that more potent and selective SARMs are needed, particularly compounds that are agonists on muscle and antagonists on prostate. The effects of androgens are augmented by functional exercise training (19), and it is possible that translation of muscle mass and strength gains induced by androgens into functional improvements may require functional exercise training. SARMs have generally well tolerated in short term trials. Larger trials of longer duration are needed to demonstrate the long-term safety and efficacy of SARMs in improving physical function and health outcomes.

\section{Designing efficient drug trials for sarcopenia and frailty}

Despite the fact that both sarcopenia and frailty are highly prevalent in older populations, a high degree of heterogeneity 


\section{PHARMACOLOGICAL INTERVENTIONS IN FRAILTY AND SARCOPENIA}

and the absence of consensus diagnostic criteria makes the design and implementation of treatment trials extremely challenging. The Task Force addressed many of these issues at its first meeting in 2012 (20). Since then, there has been some progress to define what does and does not represent sarcopenia. In 2014, Anker et al proposed "muscle wasting disease" as a new disease classification which brings together the concepts of sarcopenia, frailty, muscle wasting, and cachexia (21). This framework distinguishes acute from chronic conditions; classifies according to etiology (e.g., due to aging or an underlying medical condition), and then classifies by disease severity and progression.

\section{Defining the target population}

Patients with frailty and sarcopenia usually present with multiple chronic diseases that contribute to physical, cognitive, and functional disability. In clinical trials, this large variability increases the uncertainty in possible drug effects by inflating the confidence intervals. However, attempting to control variability by using homogeneous but less representative study populations reduces the generalizability of the results of a trial.

Recent trials have taken two general approaches to targeting patients with sarcopenia: either assessing the degree of sarcopenia and selecting those who are more severely affected or those in the middle of the spectrum; or selecting patients with specific conditions that predispose them to sarcopenia, for example, hip fracture (22). In the Lifestyle Interventions and Independence for Elders (LIFE) study, sedentary older adults were stratified according to their score on the Short Physical Performance Battery (SPPB), excluding those with scores over 9 , and oversampling those with scores of 7 or below (23). There was an overall benefit of the exercise intervention. Subgroup analysis showed that those with scores of 8 or 9 had little effect from the physical activity intervention, while a strong beneficial effect was seen in those with poorer function at baseline (scores of 7 or below). At the same time, those with lower SPPB scores are also in poorer health, resulting also in a higher rate of hospitalizations.

In terms of targeting specific conditions, the Aging in Motion (AIM) coalition (aginginmotion.org), which was established by the Alliance for Aging Research in 2011, has been working to obtain regulatory qualification for functional outcomes for clinical trials in specific conditions, including hip fracture, elective total hip arthroplasty, and hospital immobilization (Intensive Care Unit-Acquired Weakness, or ICUAW). The Task Force heard a report on one such study, a trial of LY2495655, a monoclonal antibody that targets myostatin in older individuals who were frequent fallers. Other studies have targeted patients based on age, inactivity, or presence of frailty; and patients with COPD, diabetes, heart failure, and stroke. A goal of the AIM effort is to puch for regulatory recognition of functional outcomes by including those who are functionally limited.

Whether there is a generalizable way to target sarcopenia across these conditions remains a question. The International Working Group on Sarcopenia proposed targeting patients based on an assessment of physical functioning or weakness; considering patients who are non-ambulatory or who cannot rise from a chair unassisted; or an assessment of habitual gait speed, possibly in combination with a quantitative measurement of body composition of DXA (24). The Foundation for the National Institutes of Health (FNIH) Sarcopenia Project proposed a clinical strategy to identify subjects with muscle weakness and low muscle mass (Figure 1) (25). They went on to amass clinical data from over 26,000 individuals in nine studies to conduct a cross-sectional analysis and define normal/abnormal cut-points based on a logic that a clinician would use for differential diagnosis. For example, if a subject complains he or she has functional problems in getting out of a chair, the clinician might first test to see if muscle weakness is present and, if so, whether reduced lean body mass coexists.

\section{Figure 1}

Clinical paradigm for targeting subjects with sarcopenia (reproduced after authorization) (25)

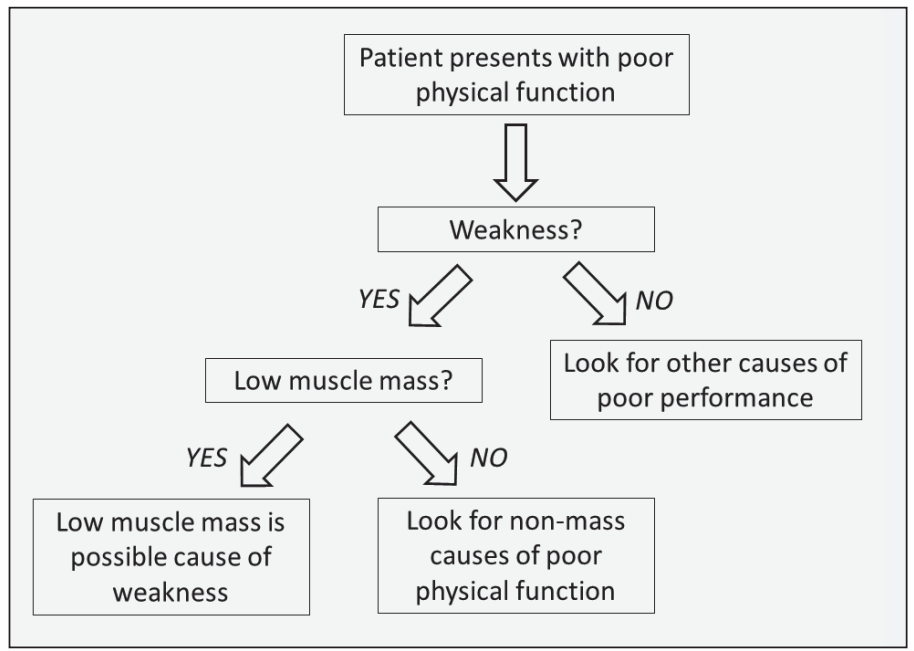

Interestingly, gender seems to be extremely relevant at modifying the relationship between different body composition parameters and physical function (26). Muscle quality, i.e., the capacity of muscle to generate force, also appears to be important, pointing to the possible need to develop criteria for muscle quality as force per unit of mass. Further studies are also needed to clarify the relationship between mass, strength, and function in diverse populations. One problem with existing data is that most of it has been obtained in high-income countries. Different screening criteria and measures may be needed in developing countries that may have limited access to imaging and other technologies. 


\section{THE JOURNAL OF FRAILTY \& AGING}

\section{Outcome measures}

\section{Physical function as a primary outcome}

Measures of physical function, particularly walking measures, have typically been used in clinical trials of sarcopenia, since walking appears to be the best predictor of disability, hospitalization, mortality, and health care expenditure (27). Several measures of walking ability have been used, including the 400 meter walk (400-MWT), 6MWD, usual gait speed test, and the SPPB which also includes a gait speed subtest. The difficulty or incapability to walk a quarter of a mile or 400 meters is also the standard measure used by the U.S. Census Bureau to assess disability. For the 400-MWT, subjects are permitted to stop but not sit or receive assistance during the walk, although a cane is allowed; and must complete the course within 15 minutes. Ability to complete the test and the time required to do so have been shown to discriminate the risk for mortality, cardiovascular disease, mobility limitation, and disability in community dwelling older adults (28).

An advantage of the 400-MWT is that there are no safety exclusions; an individual may "fail" if he or she is unable to complete the test, but failure is the outcome, rather than missing data. It also has high test-retest reliability (29). This test can therefore be used as a primary outcome measure in an intervention trial, as it was in the LIFE study. The LIFE study randomized over 1,600 sedentary individuals between the ages of 70 and 89 years to a structured moderate intensity physical activity program or a health education program for an average period of 2.6 years (23). Importantly, the use of the 400-MWT as the primary outcome enabled adjudication of the outcome even if participants were unable to come to the lab for assessment. For the LIFE study, adjudication was based on the following outcomes:

- unable to complete 400 meter walk

- unable to walk $4 \mathrm{~m}$ or unable to complete $4 \mathrm{~m}$ walk test in 10 seconds or less, i.e., gait speed less than $0.4 \mathrm{~m} / \mathrm{sec}$

- self reported inability to walk across a room without assistance

- proxy report of inability to walk across a room without assistance

- medical record documentation of inability to walk across a room (bedbound, wheelchair bound, etc.)

Using this adjudication framework, the LIFE study was able to substantially increase the amount of available information for determining the absence/presence of the studied outcomes, even among individuals who were too sick or frail to come to the clinic. In a trial without such methods, such patients would be lost to follow up, resulting in a loss of power and potentially introducing bias into the interpretation of results.

The 6MWT has been used as an outcome measures in a number of other trials, for example the Testosterone Trials (T-TRIAL) $(30,31)$. Like the 400-MWT, the 6MWT is strongly predictive of mortality (32). Other performance measures, such as the SPPB also have high prognostic value. In the LIFE pilot study, the SPPB was shown to be not only a risk factor for future health outcomes, but modifiable as well (33). Each of the different assessment tools measures different characteristics, for example performance vs. endurance; and each defines a meaningful change differently (34).

The key questions addressed at the Task Force meeting were, which measure is more efficient and what sample size is needed to demonstrate efficacy using different measures. As discussed by Espeland et al. (35), the categorization of continuous outcomes usually reduces statistical power. However, a categorical variable may still more efficiently represent an underlying continuous commonality than continuous parameters that are less directly related. Thus, for example, in the LIFE-P, analyses showed that using the 400MWT with a $20 \%$ effect size requires about 1,669 people for $80 \%$ power, compared to 5,178 subjects using the 4 -meter walk test and 4,673 using the SPPB.

Physical measures such as DXA measures of aLM and leg extension strength may also be used as either primary or secondary outcome measures, but it is unlikely that a drug indication would ever be approved for these endpoints alone since they are not directly linked to how the subject feels of functions.

\section{Other outcome measures}

Multiple secondary outcomes are also typically used in clinical trials, including additional physical performance and functional measures; changes in body composition or size; changes in nutritional status; functional changes such as a reduction in the incidence of falls, fractures, or disability; cognitive and mood changes; quality of life and health care utilization measures; and mortality. Patient-reported outcomes (PROs) have also increasingly been used as secondary outcomes to provide clinically meaningful data.

Secondary outcomes may be selected to study events that may not be widely recognized as relevant or essential to the condition. They also include those leading to a better understanding of the clinical and functional reaction of the organism to the pharmacological/non-pharmacological intervention, including adverse events. Availability of resources and time, and burden to the subject also play roles in the selection of secondary outcomes. Events/conditions that are interesting to be evaluated but may lack sufficient power to be used as primary or secondary may be referred as tertiary or exploratory outcomes.

The biological background of the disease and the phase of the study, also drive the selection of secondary outcomes. For example, secondary outcomes in early phase studies may be more focused on the kinetic and dynamic characteristics of the tested pharmacologic intervention, while in later phase studies, secondary outcomes may rather elucidate the interaction between the drug and organism as well as the clinical relevance.

Secondary outcomes may also be found embedded in the 


\section{PHARMACOLOGICAL INTERVENTIONS IN FRAILTY AND SARCOPENIA}

primary outcome. For example, a single measure of mobility such as the 400-MWT may provide information relevant to functional ability, such as the speed of completing the test, the number of stops during the conduct of the test, the speed variability, the average gait speed, etc. (36). As well, the SPPB can be deconstructed into its specific subtasks in order to obtain additional information beyond that provided by the overall score.

PROs for sarcopenia have started being incorporated into many studies of treatments for both sarcopenia and frailty. For example, in the bimagrumab studies described earlier, PROs have been included although the data have not yet been analyzed. Also in the FNIH Sarcopenia Project, a set of outcome measures have been proposed, including performance measures, PROs, health care utilization, serious injuries (e.g. fractures), and mortality. The investigators will then evaluate correlations of these measures with measures of lean mass, strength, and muscle quality. The exploration of a wide variety of secondary outcomes in this data set may provide important data regard the optimal design of future trials.

Many PROs have been proposed for measuring the frailty phenotype, yet the multidimensionality of the syndrome, as well as a serious and disturbing lack of consensus on the definition of frailty creates obvious challenges for scientific measurement. For example, Fried and colleagues have centered the definition of frailty around the cumulative effect of five criteria (largely focused on the physical aspect), while others adopted a more comprehensive approach including loss in psychological or social domains (37).

Even the five components of the frailty phenotype as defined by Fried and colleagues (38) are not included in many of the PRO measures that have been developed. Most of the measures discussed assess various physical components, while others address the psychological, cognitive, social, demographic, and health care utilization dimensions of frailty. The Irish Longitudinal Study on Aging, for example, included two multidimensional measures -- the Self-Reported Frailty Index (SRFI) and the Test-Based Frailty Index (TBFI). The investigators found that prevalence estimates varied from $11 \%$ with the SRFI to $17 \%$ with the TBFI. Interestingly, women had a higher prevalence of frailty using the SRFI compared to a lower prevalence with the TBFI, suggesting that frailty PROs may mis-estimate the prevalence of frailty in community dwelling elders and obscure gender differences.

For trials of conditions such as sarcopenia and frailty in which definitions remain unclear, flexibility is essential in selecting secondary outcomes that will enable applying results across different settings and cross-checking them according to the current different definitions and possible developments in such a dynamic field of research. Outcome measures must be selected not only based on the type of drug being tested, but also based on a clear definition of the main outcome, baseline differences in the target population, the number of sites, and the experience and training of personnel at the sites. Moreover, trialists must take into account the impact of the intervention on the studied outcome measure, which might significantly affect the duration of the trial.

\section{Biomarkers}

There is a need for biomarkers of frailty and sarcopenia aimed at improving diagnostic performance, monitor the progression of the condition(s), predict outcomes, assess treatment response, and optimize the clinical decision making process. Biomarkers should also help us better understand the relationships between aging, frailty, sarcopenia, and disability.

A complex network of biological processes influence the development of sarcopenia and frailty, including physiologic changes in metabolism, muscle strength and power, hormones, inflammatory process, and insulin resistance, among others. For example, results from the Cardiovascular Healthy Study identified increases in components of the inflammation and coagulation systems in frail compared to non-frail communitydwelling adults (39).

FRAILOMIC (www.frailomic.org) is an initiative undertaken by a consortium of university and hospitalbased research centers and the World Health Organization (WHO) to analyze multiple classical and non-classical bloodand urine-based laboratory biomarkers on samples collected from approximately 75,000 older individuals. In combination with clinical biomarkers collected from the same cohort, FRAILOMIC will identify through data mining combinations of no more than 5 biomarkers that can be used clinically for diagnosis and prognosis of disability as well as predicting the risk of frailty. Cohorts will be followed prospectively for at least 2.5 years in order to assess progression of frailty, and gender will be included in the analysis. Secondary objectives of the project include 1) assessing interactions between -omic based biomarkers and nutrition and physical exercise on the natural history of frailty, 2) testing whether the identified assessment are useful in special populations, such as people with diabetes, obesity, and cardiovascular disease; and 3) test the validity of existing frailty criteria.

\section{Novel designs}

From a statistical and study design perspective, heterogeneity in the population of individuals with sarcopenia and frailty, as well as in possible interventions, treatment effectiveness and efficacy, and clinical meaningfulness across subpopulations, combine to make clinical trial design particularly challenging. Adaptive trials have been used in other disease areas such as oncology to deal with heterogeneity, since they enable modification of multiple design elements to increase the efficiency of a trial. For sarcopenia studies, adaptive approaches may use machine learning and simulation to tailor trials for individuals with specific risk profiles at the time of randomization, such as weakness or slow gait speed, particularly when there is a specific threshold that is predictive of a downstream outcome such as disability. Eligible 


\section{THE JOURNAL OF FRAILTY \& AGING}

participants can be stratified according to their risk profiles to various interventions.

Given the complexity of such designs, Berry et al have proposed the use of platform trial designs, an extension of adaptive trial design that enables the evaluation of multiple treatments in multiple subpopulations simultaneously (40). The innovation comes from the ability to think of multiple types of trials within a single platform that handles multicomponent interventions and directly targets the effects of specific combination, enabling rapid identification of winning combinations and culling of non-efficacious arms. However, while tailoring and adapting may offer some benefits for trials of sarcopenia and frailty, unclear definitions of the conditions and at-risk populations remain obstacles to carefully consider.

\section{Overcoming barriers to clinical trial participation}

Despite the growing recognition among clinicians and scientists about the importance of frailty and sarcopenia, a lack of operational definitions for these conditions has limited their inclusion on national health policy agendas. Indeed, there have been no major studies examining the health economics of interventions for these conditions in well-defined populations. Added to this, although the vast majority of health care is delivered through primary care settings, most clinical research is carried out in specialist-oriented and hospital-based rather than primary care networks, and such research may have limited relevance to primary care processes and pathways.

A non-for-profit institute called the Foundation for Diabetes Research in Older People at Diabetes Frail (www.diabetesfrail. org) was established to address this discrepancy by focusing on primary care, where a wider number and more representative group of patients should be available for studies. The institute aims to convince primary care teams to collaborate in research studies and facilitate their involvement by creating infrastructure within primary care settings and care homes, and building the costs of primary care research into grant applications.

\section{Conclusions}

The lack of consensus definitions again arose during discussions at this Task Force meeting, as well as at previous meetings. At this point, while consensus on a definition of sarcopenia has still not been reached, agreement appears to be emerging that low mass alone provides insufficient clinical relevance if not combined with muscle weakness and/or functional impairment.

Pharmacologic and non-pharmacologic interventions for frailty and sarcopenia are nevertheless in development. The Task Force agreed on the need to build consensus on clinically meaningful functional outcomes in a more systematic manner, as well as on the need for long-term observational studies to test and validate these outcomes. In order to accomplish this, participants called for stakeholders to come together in a collaborative framework.

Conflicts of interest: Olivier Benichou is employee at Ely Lilli \& Co. Roberto Bernabei is principal investigator of an Innovative Medicines Initiative (IMI)-funded project (including partners from the European Federation of Pharmaceutical Industries and Aassociations [EFPIA]). Shalender Bhasin has received research grants from NIA, NINR, Regeneron, Lilly, and Abbvie, which are administered by the Brigham and Women's Hospital; he has served as a consultant for Abbvie, Regeneron, Sanofi, Lilly, and Viking. Matteo Cesari has received honoraria for presentations at scientific meetings and/or research fundings from Nestlé, Pfizer, Novartis and serves as workpackage leader in an IMI-funded project (including partners from EFPIA). Ronenn Roubenoff is employee at Novartis. The Gérontopôle (Chair Bruno Vellas) has received grant support from the PHRC, ANR, European Comission as well as: Abbvie, Affiris, Avid, BMS, Eisai, Elan, Envivo, Exhonit, Genentech, GSK, Ipsen, Lilly, Lundbeck, Médivation, MSD, Nutricia, Otsuka, Pharnext, Pfizer, Pierre-Fabre, Régénéron, Roche, Sanofi, Servier, TauRx Therapeutics, Wyeth. Bruno Vellas has served as consultant/advisor to Biogen, GSK, Lilly, Lundbeck, Medivation, MSD, Nestlé, Nutricia, Pfizer, Roche, Sanofi, Servier, TauRx Therapeutics, Novartis. The other authors have no conflict of interest to disclose.

Acknowledgements: This research was supported in part by the Intramural Research Program of the National Institute on Aging.

\section{References}

1. Beaudart C, Rizzoli R, Bruyère O, Reginster JY, Biver E. Sarcopenia: burden and challenges for public health. Arch Public Health. 2014;72(1):45.

2. Department of Economic and Social Affairs - Population Division. World Population Ageing, 1950-2050. New York, NY: United Nations; 2001.

3. Cruz-Jentoft AJ, Landi F, Schneider SM et al. Prevalence of and interventions for sarcopenia in ageing adults: a systematic review. Report of the International Sarcopenia Initiative (EWGSOP and IWGS). Age Ageing. 2014

4. Evans WJ, Paolisso G, Abbatecola AM et al. Frailty and muscle metabolism dysregulation in the elderly. Biogerontology. 2010;11(5):527536.

5. Cesari M, Landi F, Vellas B, Bernabei R, Marzetti E. Sarcopenia and physical frailty: two sides of the same coin. Front Aging Neurosci. 2014;6:192.

6. Ebner N, Steinbeck L, Doehner W, Anker SD, von Haehling S. Highlights from the 7th Cachexia Conference: muscle wasting pathophysiological detection and novel treatment strategies. J Cachexia Sarcopenia Muscle. 2014;5(1):27-34.

7. Lee SJ. Regulation of muscle mass by myostatin. Annu Rev Cell Dev Biol. 2004;20:61-86.

8. Davidsen PK, Gallagher IJ, Hartman JW et al. High responders to resistance exercise training demonstrate differential regulation of skeletal muscle microRNA expression. J Appl Physiol (1985). 2011;110(2):309317.

9. Hudelmaier M, Wirth W, Himmer M, Ring-Dimitriou S, Sänger A, Eckstein F. Effect of exercise intervention on thigh muscle volume and anatomical cross-sectional areas--quantitative assessment using MRI. Magn Reson Med. 2010;64(6):1713-1720.

10. Durheim MT, Slentz CA, Bateman LA, Mabe SK, Kraus WE. Relationships between exercise-induced reductions in thigh intermuscular adipose tissue, changes in lipoprotein particle size, and visceral adiposity. Am J Physiol Endocrinol Metab. 2008;295(2):E407-E412. 


\section{PHARMACOLOGICAL INTERVENTIONS IN FRAILTY AND SARCOPENIA}

11. Amato AA, Sivakumar K, Goyal N et al. Treatment of sporadic inclusion body myositis with bimagrumab. Neurology. 2014;83(24):2239-2246.

12. Rooks D. Current developments in the treatment of sarcopenia: pharmacotherapy and novel trial outcomes. J Frailty Aging. 2015;4 (Suppl 1):20 [Abstract].

13. Edwards MH, Dennison EM, Aihie Sayer A, Fielding R, Cooper C. Osteoporosis and sarcopenia in older age. Bone. 2015

14. Basaria S, Collins L, Dillon EL et al. The safety, pharmacokinetics, and effects of LGD-4033, a novel nonsteroidal oral, selective androgen receptor modulator, in healthy young men. J Gerontol A Biol Sci Med Sci. 2013;68(1):87-95.

15. Papanicolaou DA, Ather SN, Zhu $\mathrm{H}$ et al. A phase IIA randomized, placebo-controlled clinical trial to study the efficacy and safety of the selective androgen receptor modulator (SARM), MK-0773 in female participants with sarcopenia. J Nutr Health Aging. 2013;17(6):533-543.

16. Dalton JT, Taylor RP, Mohler ML, Steiner MS. Selective androgen receptor modulators for the prevention and treatment of muscle wasting associated with cancer. Curr Opin Support Palliat Care. 2013;7(4):345351.

17. Dobs AS, Boccia RV, Croot CC et al. Effects of enobosarm on muscle wasting and physical function in patients with cancer: a double-blind, randomised controlled phase 2 trial. Lancet Oncol. 2013;14(4):335-345.

18. Travison TG, Basaria S, Storer TW et al. Clinical meaningfulness of the changes in muscle performance and physical function associated with testosterone administration in older men with mobility limitation. J Gerontol A Biol Sci Med Sci. 2011;66(10):1090-1099.

19. Bhasin S, Storer TW, Berman N et al. The effects of supraphysiologic doses of testosterone on muscle size and strength in normal men. $\mathrm{N}$ Engl J Med. 1996;335(1):1-7.

20. Vellas B, Pahor M, Manini T et al. Designing Pharmaceutical Trials for Sarcopenia in Frail Older Adults: EU/US Task Force Recommendations. J Nutr Health Aging. 2013;17(7):612-618.

21. Anker SD, Coats AJ, Morley JE et al. Muscle wasting disease: a proposal for a new disease classification. J Cachexia Sarcopenia Muscle. 2014;5(1):1-3.

22. Vellas B, Fielding R, Miller R et al. Designing drug trials for sarcopenia in older adults with hip fracture. A task force from the International Conference on Frailty and Sarcopenia Research (ICFSR). J Frailty Aging. 2014;3 (4):199-204.

23. Pahor M, Guralnik JM, Ambrosius WT et al. Effect of Structured Physical Activity on Prevention of Major Mobility Disability in Older Adults: The LIFE Study Randomized Clinical Trial. JAMA. 2014;311(23):2387-2396.

24. Fielding RA, Vellas B, Evans WJ et al. Sarcopenia: an undiagnosed condition in older adults. Current consensus definition: prevalence, etiology, and consequences. International working group on sarcopenia. J Am Med Dir Assoc. 2011;12(4):249-256.

25. Studenski SA, Peters KW, Alley DE et al. The FNIH Sarcopenia Project: Rationale, Study Description, Conference Recommendations, and Final Estimates. J Gerontol A Biol Sci Med Sci. 2014;69(5):547-558.
26. Cesari M, Rolland Y, Abellan Van Kan G, Bandinelli S, Vellas B Ferrucci L. Sarcopenia-Related Parameters and Incident Disability in Older Persons: Results From the "Invecchiare in Chianti" Study. J Gerontol A Biol Sci Med Sci. 2015;70 (4):547-558.

27. Hardy SE, Kang Y, Studenski S, Degenholtz HB. Ability to walk $1 / 4$ mile predicts subsequent disability, mortality, and health care costs. J Gen Intern Med. 2011;26(2):130-135.

28. Newman AB, Simonsick EM, Naydeck BL et al. Association of longdistance corridor walk performance with mortality, cardiovascular disease, mobility limitation, and disability. JAMA. 2006;295(17):2018-2026.

29. Rolland Y, Lauwers-Cances V, Pahor M, Fillaux J, Grandjean H, Vellas B. Muscle strength in obese elderly women: effect of recreational physical activity in a cross-sectional study. Am J Clin Nutr. 2004;79(4):552-557.

30. Snyder PJ, Ellenberg SS, Cunningham GR et al. The Testosterone Trials: Seven coordinated trials of testosterone treatment in elderly men. Clin Trials. 2014;11(3):362-375.

31. Cunningham GR, Stephens-Shields AJ, Rosen RC et al. Association of sex hormones with sexual function, vitality, and physical function of symptomatic older men with low testosterone levels at baseline in the testosterone trials. J Clin Endocrinol Metab. 2015;100(3):1146-1155.

32. Mutikainen S, Rantanen T, Alén M et al. Walking ability and all-cause mortality in older women. Int J Sports Med. 2011;32(3):216-222.

33. Pahor M, Blair SN, Espeland M et al. Effects of a physical activity intervention on measures of physical performance: Results of the lifestyle interventions and independence for Elders Pilot (LIFE-P) study. J Gerontol A Biol Sci Med Sci. 2006;61(11):1157-1165.

34. Perera S, Mody S, Woodman RC, Studenski S. Meaningful change and responsiveness in common physical performance measures in older adults. J Am Geriatr Soc. 2006;54(5):743-749.

35. Espeland MA, Gill TM, Guralnik J et al. Designing clinical trials of interventions for mobility disability: results from the lifestyle interventions and independence for elders pilot (LIFE-P) trial. J Gerontol A Biol Sci Med Sci. 2007;62(11):1237-1243.

36. Vestergaard S, Patel KV, Bandinelli S, Ferrucci L, Guralnik JM Characteristics of 400-meter walk test performance and subsequent mortality in older adults. Rejuvenation Res. 2009;12(3):177-184.

37. Walston J, Hadley E, Ferrucci L et al. Research agenda for frailty in older adults: toward a better understanding of physiology and etiology: summary from the American Geriatrics Society/National Institute on Aging Research Conference on Frailty in Older Adults. J Am Geriatr Soc. 2006;54(6):991-1001.

38. Fried LP, Tangen CM, Walston J et al. Frailty in older adults: evidence for a phenotype. J Gerontol A Biol Sci Med Sci. 2001;56(3):M146-M156.

39. Walston J, McBurnie MA, Newman AB et al. Frailty and activation of the inflammation and coagulation systems with and without clinical comorbidities: results from the Cardiovascular Health Study. Arch Intern Med. 2002;162(20):2333-2341.

40. Berry SM, Connor JT, Lewis RJ. The platform trial: an efficient strategy for evaluating multiple treatments. JAMA. 2015;313(16):1619-1620. 